\title{
MODELING STOCHASTIC LEAD TIMES IN MULTI-ECHELON SYSTEMS
}

\author{
E. B. Diks \\ Department of Mathematics and Computing Science \\ Eindhoven University of Technology, P.O. Box 513 \\ $5600 \mathrm{MB}$ Eindhoven, The Netherlands \\ M. C. VAN DER HEIJDEN \\ Department of Technology and Management \\ University of Twente, P.O. Box 217 \\ 7500 AE Enschede, The Netherlands
}

\begin{abstract}
In many multi-echelon inventory systems, the lead times are random variables. A common and reasonable assumption in most models is that replenishment orders do not cross, which implies that successive lead times are correlated. However, the process that generates such lead times is usually not well defined, which is especially a problem for simulation modeling. In this paper, we use results from queuing theory to define a set of simple lead time processes guaranteeing that (a) orders do not cross and (b) prespecified means and variances of all lead times in the multiechelon system are attained.
\end{abstract}

\section{INTRODUCTION}

The fact that the lead time in an inventory system is rarely constant is widely recognized. It is important to account for lead time variability when analyzing multiechelon inventory systems, because ignoring it may lead to a high cost and a poor performance (cf. Gross and Soriano [8]). An important issue when incorporating stochastic lead times is whether successive lead times are independent, because in that case orders can cross. A crossover occurs when a quantity that was ordered in a 
latter period arrives before one that was ordered in an earlier period. In practice, successive lead times are usually dependent (cf. Yano [21]); therefore, the "no crossing" assumption needs to be incorporated in the model. Various modeling approaches have been used to circumvent this complication. When the interval between successive orders is large enough, the probability of crossover is negligible and can be omitted (cf. Hadley and Whitin [9]). Friedman [7] circumvented the problem by assuming that there is always only one order outstanding. In Sphicas [16] and.Sphicas and Nasri [17], the problem of crossovers is eliminated by assuming that unit demands are noninterchangeable. It is clear that these approaches are not generally valid.

To our knowledge, Kaplan [12] was the first that explicitly incorporated the no crossing assumption. A mechanism of the arrival of orders is used, to ensure that orders never cross, whereas in general lead times will be dependent. Kaplan (see also Nahmias [15] and Anupindi, Morton, and Pentico [2]) developed this mechanism for a periodic review system, which places an order at the beginning of every period. This mechanism is based on a stationary, discrete-time Markov process $\{U(t)\}$ with nonnegative-integer states, representing the number of outstanding orders after a possible delivery at time $t$, with

$$
\operatorname{Pr}\{U(t+1)=j \mid U(t)=i\}= \begin{cases}p_{j}, & j=0,1, \ldots, i \\ 1-\sum_{n=0}^{i} p_{n}, & j=i+1\end{cases}
$$

The situation where at time $t+1$ no delivery is made corresponds with $j=i+1$. Notice that Eq. (1) assumes that the probability of arrival of an outstanding order is independent of the number of outstanding orders. Ehrhardt [5] derived the distribution of the lead time $L$, for this specification, namely,

$$
\operatorname{Pr}\{L=i\}= \begin{cases}p_{0}, & i=0, \\ \left(1-p_{0}\right)\left(1-p_{0}-p_{1}\right) \ldots\left(1-\sum_{j=0}^{i-1} p_{j}\right) \sum_{j=0}^{i} p_{j}, & i=1,2, \ldots, m,\end{cases}
$$

where $m$ denotes the maximum lead time. Like Zipkin [22], we think it is hard to imagine a physical process giving rise to this scenario. Moreover, many parameters need to be estimated from data and, furthermore, Zipkin showed that not any lead time distribution (e.g., the geometric distribution) can be attained. Zipkin extended the arrival mechanism of Kaplan [12] by relaxing Eq. (1) (along with the discrete time assumption). However, it is not clear how to choose an order arrival process that fits certain lead time characteristics (the mean and variance as observed in practice).

Heuts and de Klein [11] introduced another model to deal with stochastic lead times. They distinguish between a start-up and a follow-up order. For a start-up order $\operatorname{Pr}\left\{L=l_{j}\right\}=p_{j}$, where the discrete lead time $L$ is defined at the points $l_{0}, l_{1}, \ldots, l_{M-1}$ (in ascending order). A follow-up order will be delivered simulta- 
neously with the start-up order not yet arrived. However, their model requires a large amount of information (i.e., all the possible lead times $l_{j}$ of the start-up order and its probabilities $p_{j}$ ), whereas our model only requires the first two moments of the lead time (and possibly the first-order autocorrelation).

In this paper, we develop a simple model for a lead time process in which orders do not cross. We show that the combination of a single-server queue and a deterministic pipeline yields a versatile and simple lead time process. We use results from queuing theory to choose the parameters of this process such that the successive throughput times (approximately) match a prespecified lead time mean and variance. Although the approach is general applicable, we focus on divergent networks with a periodic review, order-up-to policy. The results of this paper facilitate building a simulation model of multi-echelon inventory systems, to be used for scenario analysis and/or validation of approximate analytical methods. In fact, we developed this approach for the latter purpose (see van der Heijden, Diks, and de Kok [10]).

The remainder of this paper is structured as follows. First, we consider a singlelocation inventory system. Section 2 deals with the model and approach for this situation. The analysis of the model is discussed in Sections 3 and 4. In Section 5, we show how the lead time model can be used in a general multi-echelon setting. In Section 6, the approximations are validated for divergent networks under periodic review by a simulation study. Finally, we give some concluding remarks in Section 7.

\section{SINGLE-LOCATION INVENTORY SYSTEM}

Consider a single-location inventory system in which the lead time $L$ is a random variable with mean $\mu_{L}$ and variance $\sigma_{L}^{2}$. Denote by $A_{n}$ the time period between issuing the $(n-1)$ st and the $n$th replenishment order. $A_{n}$ can be a random variable (e.g., under continuous review) or deterministic (e.g., under periodic review). Because it is assumed that orders cannot cross, the subsequent lead times faced in the inventory system are correlated for most lead time distributions. Therefore, it becomes rather cumbersome to model a lead time process for which the mean and variance coincide with the target values $\mu_{L}$ and $\sigma_{L}^{2}$, respectively.

To illustrate this, we consider a single-location inventory system under periodic review. Figure 1 depicts the arrival times of two subsequent orders. At time $t$, an order is placed at the supplier with lead time $L_{1}$. This order arrives at $t+L_{1}$. At time $t+R$, the stockpoint again inspects the inventory position and places an order with

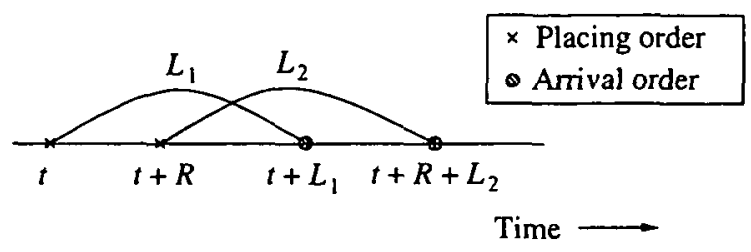

Figure 1. Arrival process of orders. 
lead time $L_{2}$. This order arrives at $t+R+L_{2}$. Because subsequent orders do not cross, there has to hold $R+L_{2} \geq L_{1}$. If the lead time distribution of $L$ is such that this always holds, the successive lead times can be independent. An example of such a distribution is a uniform distribution on $[\alpha, \alpha+\beta]$, with $\alpha \geq 0$ and $0 \leq \beta \leq R$. For the more general case where not always does $R+L_{2} \geq L_{1}$, it is obvious that $L_{2}$ depends on $L_{1}$. In the remainder of this paper, we develop an approach to model the lead time process of interrelated lead times, such that the mean and variance of the lead time equals the predetermined $\mu_{L}$ and $\sigma_{L}^{2}$, respectively.

\subsection{Model and Approach}

The lead time is modeled by the sojourn time in a GI/G/1 queue plus a deterministic pipeline time. The arrival of a customer at this queue corresponds to the stockpoint placing an order and, because the server adopts the first come-first served discipline, customers (i.e., orders) cannot cross. For our convenience, we introduce the following notation:

$\mu_{X}:=$ the mean of random variable $X$,

$\sigma_{X}:=$ the variance of random variable $X$,

$c_{X}:=$ the coefficient of variation of a random variable $X, c_{X}=\sigma_{X} / \mu_{X}$,

$\mu_{3}[X]:=$ the third central moment of random variable $X, \mu_{3}[X]=E\left[X-\mu_{X}\right]^{3}$,

$F_{X}:=$ the cumulative distribution function of a random variable $X$,

$\lambda:=$ the arrival rate at the queuing system,

$B:=$ the service time of a customer in the queuing system,

$W:=$ the waiting time of a customer in the queueing system,

$S:=$ the sojourn time of a customer in the queuing system, $S=W+B$.

The lead time is not modeled solely by a queuing system for the following reason. In practice, the mean lead time can be large in contrast to its small variance. To obtain a lead time process for which the sojourn time has a large mean and a small variance, a very high utilization $(>0.999)$ is required. So, using the sojourn time of the GI/G/1 queue solely is inadequate; therefore, we suggest to model the lead time as the sum of the sojourn time in a GI/G/1 queue plus a fixed time in a pipeline (see Fig. 2).

By setting this fixed time properly, we avoid lead time processes with an extremely high utilization. Thus, the lead time $L$ is given by

$$
L:=l+S,
$$

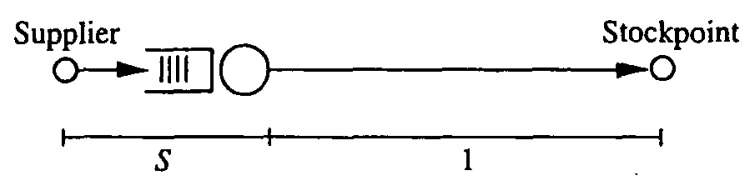

FigUre 2. Model of the lead time process. 
where $l$ denotes a fixed nonnegative time and the random variable $S$ denotes the stochastic part of the lead time (in general, it depends on preceding lead times). As one possible interpretation, one might think of $l$ as a fixed handling and transportation time and $S$ as the production time, which is subjected to capacity constraints.

The sojourn time of the GI/G/1 queue plus the deterministic pipeline time has mean $\mu_{W}+\mu_{B}+l$ and variance $\sigma_{W}^{2}+\sigma_{B}^{2}$. So, we have to find the parameters of the lead time process, such that $\mu_{L}=\mu_{W}+\mu_{B}+l$ and $\sigma_{L}^{2}=\sigma_{B}^{2}+\sigma_{W}^{2}$ for given $\mu_{L}$ and $\sigma_{L}^{2}$. The process by which replenishment orders are generated is given, so the remaining degrees of freedom are $l$ and the service distribution $B$. Using only the first two moments of $B$ to analyze the GI/G/1 queue, this means that we have to find the right values for $l, \mu_{B}$, and $\sigma_{B}^{2}$. So we have one excess degree of freedom. We deal with this as follows. First, we show how to determine the values of $\mu_{B}$ and $\sigma_{B}^{2}$ for a given value of $l$ (Section 3). Next, we discuss a proper choïce of $l$ (Section 4).

\section{DETERMINATION OF $\mu_{B}$ AND $\sigma_{B}^{2}$}

The objective of many papers in the queueing literature is to determine performance characteristics (e.g., mean and variance of sojourn time) given information regarding the arrival process and the service process. In this section, we have to deal with the "inverse" problem: determine the service process characteristics given information regarding the arrival process and the mean and variance of the sojourn time. Specifically, determine $\mu_{B}$ and $\sigma_{B}^{2}$ such that $\mu_{B}+\mu_{W}=\mu_{L}-l$ and $\sigma_{B}^{2}+\sigma_{W}^{2}=$ $\sigma_{L}^{2}$. To solve this problem, we developed an algorithm that uses some techniques developed in the literature. First, we briefly address these queuing techniques in Section 3.1. Next, we discuss in Section 3.2 a nested bisection algorithm to determine $\mu_{B}$ and $\sigma_{B}^{2}$.

\subsection{Queuing Approximations}

In this section, we address several techniques that determine $\mu_{W}$ and $\sigma_{W}^{2}$ from $\mu_{B}$ and $\sigma_{B}^{2}$. We distinguish between the $D / G / 1$ queue (Section 3.1.1) and the GI/G/1 queue (Section 3.1.2), because for the former queue good approximations have been developed and because it represents a specific case, namely, the periodic review.

\section{1. 1. $D / G / 1$ model.3}

Fredericks [6] and Bhat [3] (F\&B)

In Fredericks [6], an excellent approximation is given for the mean waiting time, denoted by $\mu_{W}$, in a $D / G / 1$ queue:

$$
\mu_{W}=\frac{\int_{R}^{\infty}(t-R) d F_{B}(t)}{F_{B}(R)-1+\int_{R}^{\infty} e^{\delta(t-R)} d F_{B}(t)},
$$


where $\delta$ is the unique solution to the equation

$$
e^{-\delta R} \int_{0}^{\infty} e^{\delta y} d F_{B}(y)=1
$$

In Whitt [20], several diffusion approximations are given for $\mu_{W}$ in case of heavy traffic (say, $\rho=\lambda \mu_{B}>0.95$ ). Bhat [3] gave an approximation for $\sigma_{W}^{2}$ :

$$
\sigma_{W}^{2}=z_{1}^{2}+\frac{z_{2}}{3(1-\rho)}, \quad \text { where } z_{1}:=\frac{\lambda \sigma_{B}^{2}}{2(1-\rho)}, \quad z_{2}:=\lambda \mu_{3}[B] .
$$

$\mu_{3}[B]$ in Eq. (3) is determined by first fitting a mixture of two Erlang distributions to the first two moments of $B$ (cf. Tijms [18]) and next computing its third moment.

de Kok [13]

Let, for $n=0,1,2, \ldots$, the random variables $B_{n}$ and $W_{n}$ denote the service time and the waiting time of the $n$th customer, respectively. Furthermore, suppose that the 0 th customer arrives at an empty system, so that $W_{0}=0$. Then, it is easily seen that the following relation holds:

$$
W_{n}=\left(W_{n-1}+B_{n-1}-R\right)^{+}, \quad n=1,2, \ldots,
$$

where $x^{+}:=\max (0, x)$. The moment-iteration method developed by de Kok [13] determines the distribution of $W_{n}$ by an iterative procedure. It yields excellent results. Only for the case of heavy traffic $(\rho>0.95)$ do we suggest the use of the F\&B approach.

In case of discrete lead times, one might use the simple method of Adan, van Eenige, and Resing [1], which fits a discrete distribution to $B$. Next, they used the moment-iteration method for the $D / G / 1$ queue to obtain $\mu_{W}$ and $\sigma_{W}^{2}$. Numerical results show excellent performance of the method.

3.1.2. GI/G/1 model. As a GI/G/1 analogue of the F\&B approach of Section 3.1.1, we suggest using the approximation of Krämer and Langenbach-Belz [14] to approximate $\mu_{W}$ and again approximate $\sigma_{W}^{2}$ by the formula of Bhat [3] $\left(z_{1}\right.$ and $z_{2}$ of Eq. (3) need to be adapted). As mentioned by Tijms [18], the approximation of Krämer and Langenbach-Belz [14] is a very useful and quick estimate when $c_{A}$ is not too large. Also, we could use the moment-iteration method of de Kok [13] for the GI/G/1 queue to determine $\mu_{W}$ and $\sigma_{W}^{2}$ (Eq. (4) needs to be adapted).

\subsection{Algorithm}

Without loss of generality, it is assumed that the review period $R$ equals 1 , that is, $\lambda=1$. By applying either the F\&B approximations or the moment-iteration algorithm of de Kok [13], we are able to compute $\mu_{S}\left(\mu_{B}, \sigma_{B}^{2}\right)$ and $\sigma_{S}^{2}\left(\mu_{B}, \sigma_{B}^{2}\right)$, denoting the mean and variance of the sojoum time given $\mu_{B}$ and $\sigma_{B}^{2}$, respectively. To explain our algorithm (which determines $\mu_{B}$ and $\sigma_{B}^{2}$ such that $\mu_{S}=\mu_{L}-l$ and $\sigma_{S}^{2}=\sigma_{L}^{2}$ ), we depicted the behavior of both functions in Figure 3 (using the moment-iteration method). 

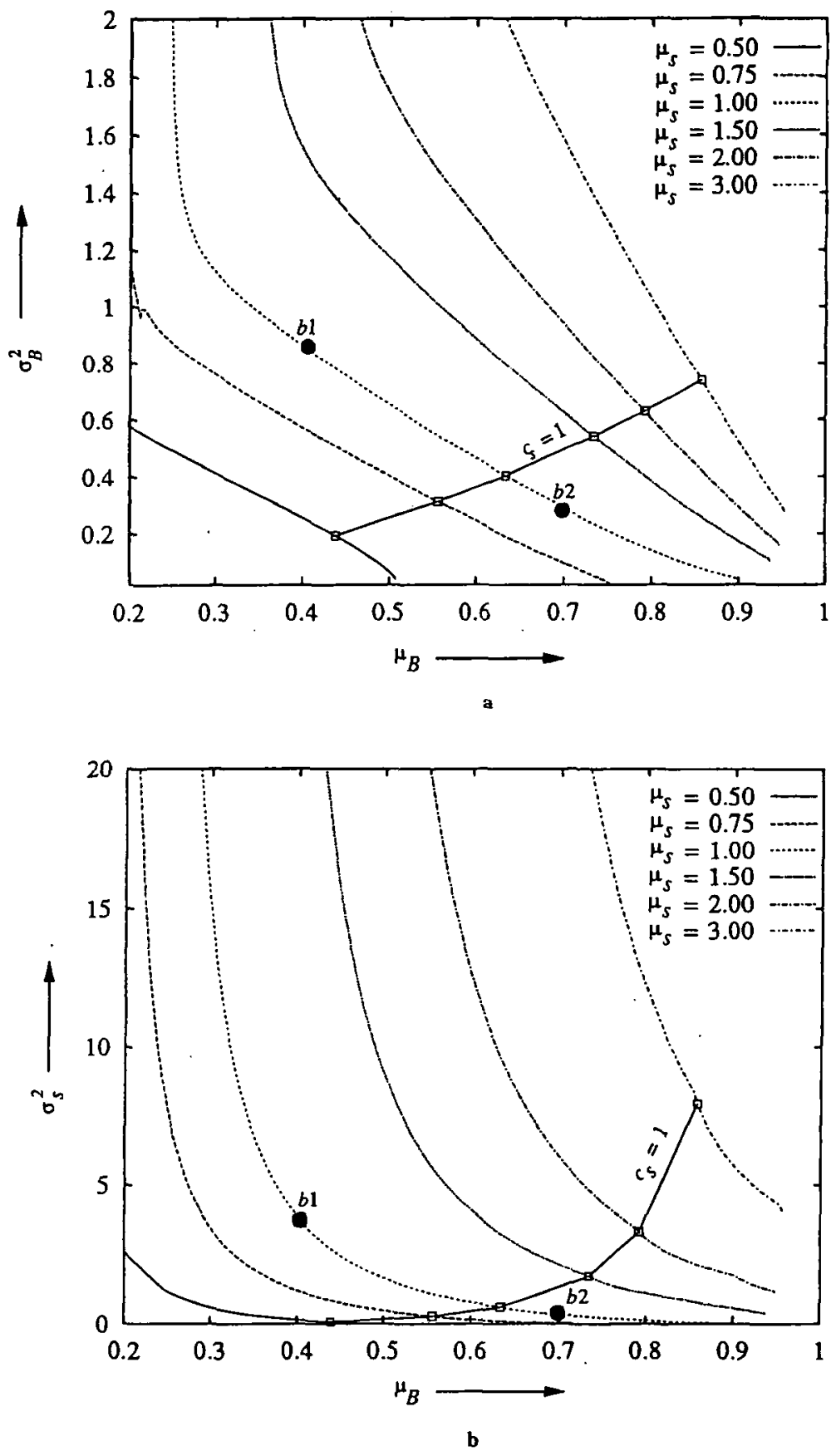

Figure 3. Behavior of $\sigma_{B}^{2}$ (a) and $\sigma_{S}^{2}$ (b) in a D/G/1 queue as a function of $\mu_{B}$. 
Figure 3a shows for which $\left(\mu_{B}, \sigma_{B}^{2}\right)$ a certain mean sojourn time $\mu_{S}$ is achieved. It indicates that when $\mu_{B}$ decreases, $\sigma_{B}^{2}$ at first increases linearly but, after a certain point, increases more than linear. Figure $3 \mathrm{~b}$ depicts the behavior of the variance $\sigma_{S}^{2}$ for all the points depicted in Figure $3 a$.

Let us explain the algorithm by considering an example. Suppose $\mu_{S}=1$ and $\sigma_{S}^{2}=1$. First, we choose a mean service time, say, 0.4 , and determine by bisection how to set $\sigma_{B}^{2}$ such that $\mu_{S}=1$. In Figure 3, this point is depicted by $b 1$. Because $\sigma_{S}^{2}$ in $b 1$ exceeds 1 , we decide to increase $\mu_{B}$, say, to 0.7 . For this service time, again the $\sigma_{B}^{2}$ is determined such that $\mu_{S}=1$. This yields point $b 2$. However, because $\sigma_{S}^{2}$ in $b 2$ is too small we now decrease $\mu_{B}$. Proceed with this procedure until the point $\left(\mu_{B}, \sigma_{B}^{2}\right)$, for which $\mu_{S}=1$ and $\sigma_{S}^{2}=1$ is found. This nested bisection procedure is formalized in the Appendix.

\section{DETERMINATION OF I}

As described in Section 2.1, it is important to choose a proper $l$. In this section, we give two methods to split the lead time into the fixed part $l$ and random part $S$. Again, we distinguish between the D/G/1 model (Section 4.1) and the GI/G/1 model (Section 4.2).

\section{1. $D / G / 1$ Model}

The first method assumes that there is some knowledge on the correlation of successive lead times. Specifically, this method determines how $l$ should be set such that the first-order autocorrelation equals $\rho_{L}$. Using Eq. (2), it follows that

$$
\rho_{L}=\frac{\operatorname{cov}\left(S_{1}, S_{2}\right)^{-}}{\sigma_{S}^{2}}
$$

where $S_{1}$ and $S_{2}$ are two successive sojoum times of the D/G/1 queue. By conditioning on $S_{1}$, it can be shown that

$$
\operatorname{cov}\left(S_{1}, S_{2}\right)=\sigma_{S}^{2}-\left(R-\mu_{B}\right) \mu_{S}+\int_{0}^{R}\left(R x-x^{2}\right) d F_{S}(x) .
$$

Substituting Eq. (6) in Eq. (5) yields

$$
\rho_{L}=1-\frac{\left(R-\mu_{B}\right) \mu_{S}-\int_{0}^{R}\left(R x-x^{2}\right) d F_{S}(x)}{\sigma_{S}^{2}} .
$$

By fitting a suitable distribution on $S$, we are able to actually compute $\rho_{L}$. As described in Tijms [18], we fit a mixture of two Erlang distributions on its mean and variance.

From Eq. (7), it follows that when $\sigma_{S}^{2}$ is fixed and $\mu_{S}$ converges to 0 , then $\rho_{L}$ eventually converges to 1 . This is intuitively clear. From $\mu_{B}<\mu_{S}$, it follows that when $\mu_{S}$ converges to $0, \mu_{B}$ also converges to 0 . To still meet the fixed variance $\sigma_{S}^{2}$, the 
$\sigma_{B}^{2}$ increases considerably when $\mu_{B}$ converges to 0 . So, for a small $\mu_{S}$, the $c_{B}$ is very large. This results in a "lumpy" service time distribution; that is, most of the time $B$ is small, but on rare occasions $B$ is huge. Hence, the sojourn time of a customer becomes highly correlated with the sojourn time of its preceding customer.

On the other hand, when $\sigma_{S}^{2}$ is fixed and $\mu_{S}$ is very large (with respect to $\sigma_{S}^{2}$ ), then $\rho_{L}$ is also large. Also, this is intuitively clear. When $\mu_{S}$ is large compared to $\sigma_{S}^{2}$, the $c_{S}$ is small. Hence, the behavior of $S$ is more or less deterministic.

Figure 4 depicts the behavior of $\rho_{L}$ as a function of $\mu_{S}$ in a periodic review system with $R=1$. As we expected, this figure shows that $\rho_{L}$ is approximately 1 for a small $\mu_{s}$ and large for $\mu_{5}$ sufficiently large. Furthermore, this figure shows that not any $\rho_{L}$ can be obtained for given $\mu_{L}$ and $\sigma_{L}^{2}$. The smaller $\sigma_{L}^{2}$ is, the larger the range of $\rho_{L}$ becomes. For some values of $\rho_{L}$, there even are two ways of splitting the lead time. In advance, it is not clear which of these two possibilities yields the best way of splitting the lead time. Finally, we note that $\rho_{L}$ is independent of $\mu_{L}$ but only depends on $\mu_{S}\left(\leq \mu_{L}\right)$ and $\sigma_{L}^{2}$.

The second method, which yields a suitable way of splitting $L$ into the fixed $l$ and the random $S$, is just by taking an exponential distribution for $B$. Then from queuing theory it follows that the sojoum time in a $D / M / 1$ queue is exponentially distributed as well. So $c_{S}=1$. After some elementary algebra, it follows that

$$
l=\left(1-c_{L}\right) \mu_{L}, \quad c_{L} \leq 1 .
$$

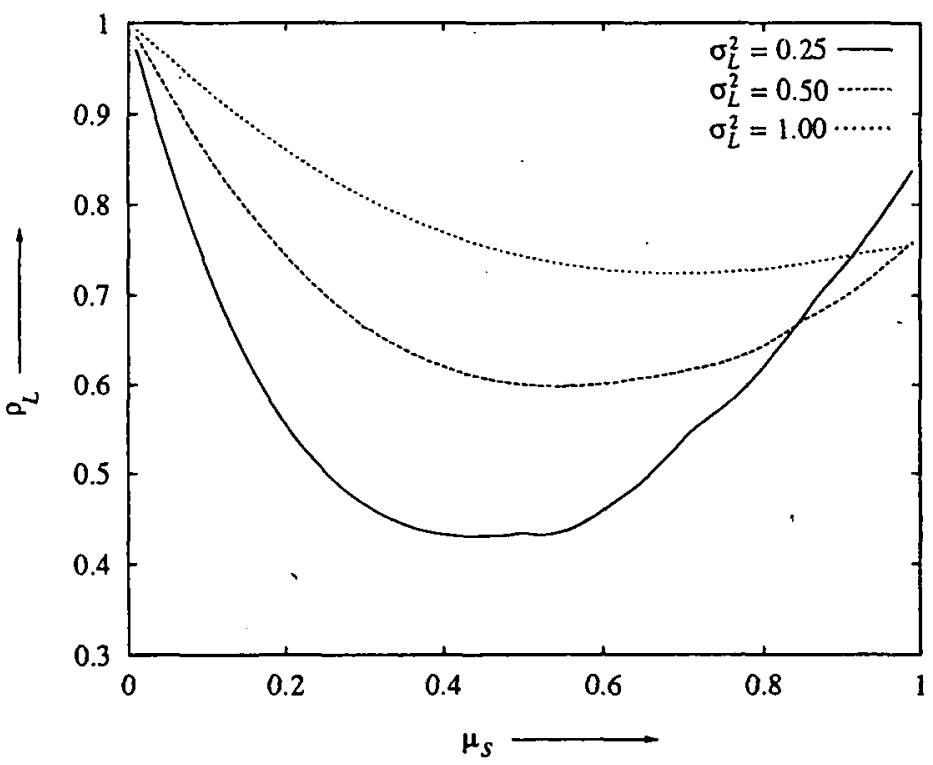

Figure 4. Behavior of $\rho_{L}$ as a function of $\mu_{S}$, when $R=1$. 
Figure 3a shows indeed that all points $\left(\mu_{B}, \sigma_{B}^{2}\right)$ with $c_{S}=1$ are on the line for which $c_{B}=1$. So, by using the splitting rule of Eq. (8), the service process easily follows from the analysis of the $D / M / 1$ queue: $B$ is exponentially distributed with

$$
\mu_{B}=\mu_{S}\left(1-e^{-\frac{1}{\mu_{S}}}\right)
$$

\subsection{GI/G/1 Model}

As in Section 4.1, we address the same two methods of splitting $L$. For applying the first method, we need to have an expression for the covariance of two successive sojourn times. Unlike the $D / G / 1$ queue, the variability of the arrival process of the GI/G/1 queue has to be taken into account. So, Eq. (6) slightly changes:

$$
\operatorname{cov}\left(S_{1}, S_{2}\right)=\sigma_{S}^{2}-\left(\mu_{A}-\mu_{B}\right) \mu_{S}+\int_{0}^{\infty} \int_{0}^{\infty} x\left(1-F_{A}(r+x)\right) d r d F_{S}(x)
$$

By fitting a mixture of two Erlang distributions on the mean and variance of $A$, we can obtain a more tractable expression for Eq. (10). This means that the interarrival time $A$ follows an $E_{k_{1}, \theta_{1}}$ distribution with probability $\alpha$ and an $E_{k_{2}, \theta_{2}}$ distribution with probability $1-\alpha$. The parameters $\alpha, k_{1}, k_{2}, \theta_{1}$, and $\theta_{2}$ can easily be obtained from $\mu_{A}$ and $\sigma_{A}^{2}$. For more details regarding the fitting procedure, we refer to Tijms [18]. After some algebra, Eq. (10) is elaborated as

$$
\begin{aligned}
\operatorname{cov}\left(S_{1}, S_{2}\right)= & \sigma_{S}^{2}-\left(\mu_{A}-\mu_{B}\right) \mu_{S} \\
& +\sum_{i=1}^{2} \frac{\alpha_{i}}{\theta_{i}} \sum_{j=0}^{k_{i}-1} \sum_{l=0}^{j} \frac{\theta_{i}^{l}}{l !} \int_{0}^{\infty} x^{l+1} e^{-\theta_{i} x} d F_{S}(x) .
\end{aligned}
$$

Also on $S$ we fit a mixture of two Erlang distributions so as to be able to compute $\rho_{L}$.

Again, the second method can be applied. Similar results hold, because the distribution of the sojourn time in a GI/M/1 queue is exponentially distributed. So the service time $B$ is exponentially distributed with

$$
\mu_{B}=\mu_{S}\left(1-A^{*}\left(\frac{1}{\mu_{S}}\right)\right)
$$

where $A^{*}(s)$ denotes the Laplace-Stieltjes transform of $A$. Note that for the aforementioned mixture of two Erlang distributions we have:

$$
A^{*}(s)=\alpha\left(\frac{\theta_{1}}{\theta_{1}+s}\right)^{k_{1}}+(1-\alpha)\left(\frac{\theta_{2}}{\theta_{2}+s}\right)^{k_{2}}
$$

\section{MULTI-ECHELON SYSTEMS}

In this section, we extend the results of Section 2 to arbitrary large networks of stockpoints. We distinguish between push systems (Section 5.1) and pull systems 
(Section 5.2). By "push," we mean that each stockpoint ships replenishment orders to its successors immediately upon arrival of a shipment, whereas "pull" reflects that the receiving stockpoint issues replenishment orders.

\subsection{Push Systems}

In a convergent system, every stockpoint has a unique successor (but may have several predecessors), whereas in a divergent system every stockpoint has a unique predecessor (but may have several successors). Several papers concerning divergent systems use a push mechanism (see van der Heijden et al. [10]). Upon arrival of an order at a stockpoint, the products are shipped toward its successors. The D/G/1 analysis performed in Sections 3 and 4 applies to the most upstream stockpoint if a periodic review policy is used. When the upstream stockpoint uses a continuous review policy, the GI/G/1 model may be applied. The arrival process of a downstream stockpoint is determined by the departure process of its upstream predecessor. Hence, the interarrival times generally will be correlated. As an approximation, we suggest to model the lead time process in a downstream stockpoint by the sojourn time in a GI/G/1 queue plus a fixed handling and transportation time. So we disregard the dependency between the interarrival times. In Section 6, this assumption is verified by simulation.

For our convenience, we describe how to model the lead time process of the downstream stockpoint in a two-stage serial system (see Fig. 5). The extension to larger serial systems, or even divergent systems, is straightforward, because for all cases the arrival process of the GI/G/1 queue equals the departure process of its predecessor. We use a two-moment approximation for the interdeparture time process. It is clear that the mean interdeparture time equals the mean interarrival time at stockpoint 1 . The variance of the interdeparture time of stockpoint 1 can be determined from the three approximations for the squared coefficient of variation of the interdeparture times of a GI/G/1 queue given by Buzacott and Shanthikumar [4].

For convergent systems, the push mechanism is hardly used, because it is mainly used to model an assembly system. Then material coordination is required, because it does not make sense to ship parts to its successor if not all the parts are available. Hence, a push mechanism is not appropriate. Moreover, in a push mechanism, the arrival process of a stockpoint equals the superposition of all the departure processes of its predecessors. It is doubtful whether this arrival process can be described appropriately by independent arrivals where its interarrival times are only based on the first two moments. Hence, for these systems an alternative model of the lead times probably is required.

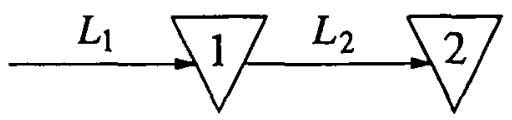

Figure 5. The two-serial system. 


\subsection{Pull Systems}

In pull systems, a stockpoint typically places an order at all its upstream stockpoints when its inventory position drops below a certain reorder point. When one of these upstream stockpoints is not able to satisfy the order immediately, then (a) this order is lost or (b) it is backordered and delivered as soon as possible. In the first case, some lead times may represent an order of amount zero. Like Kaplan [12], we adopt the convention that such an order can be treated as a pseudo-order or infinitesimal amount but to be considered as outstanding until the pseudo-order arrives. Hence, for every stockpoint the $D / G / 1$ model may be applied in case of periodic review, and the GI/G/1 model may be used as an approximation in case of continuous review. A subject for further research is to determine for which inventory policies this yields satisfactory results. However, for the second case the lead time process of a stockpoint also depends on the waiting times at its upstream stockpoints. In that case, it is hard to characterize the arrival process at this stockpoint.

\section{NUMERICAL RESULTS}

Let us first address the numerical results with respect to a single inventory system (Section 6.1). Next, we examine the approximation of assuming independent arrivals of orders at downstream stockpoints (see Section 6.2).

\subsection{Single-Location Inventory System}

Consider a single-location inventory system. Because the splitting rule of Eq. (8) yields exact results, there is no need for testing. For the case where the lead time is split so as to attain a predetermined first-order autocorrelation $\rho_{L}$, we examined several instances of a single location inventory system under periodic review. Again, without loss of generality $R:=1$.

Table 1 shows the results when the target mean lead time $\mu_{L}$ equals 2 . We considered three different $\sigma_{L}^{2}$. For $\sigma_{L}^{2}=0.25$ we require $\rho \in\{0.5,0.65\}$, for $\sigma_{L}^{2}=0.5$ we require $\rho_{L} \in\{0.7,0.8\}$, and for $\sigma_{L}^{2}=1$ we require $\rho_{L}=0.8$. For every instance, we determined both possibilities (see Fig. 4) of attaining $\rho_{L}$, denoted by 1 and 2 . Furthermore, we compare the $\mathrm{F} \& \mathrm{~B}$ technique and the moment-iteration method of de Kok [13]. In Table $1, \hat{\mu}_{L}, \hat{\sigma}_{L}$, and $\hat{\rho}_{L}$ denote the simulated values. From Table 1 , it is clear that for 8 out of the 20 cases the results are satisfactory (i.e., $\hat{\rho}_{L}$ deviates from $\rho_{L}$ maximally by 0.04 ). However, for the worst 7 cases, the results are very poor (i.e., $\hat{\rho}_{L}$ deviates from $\rho_{L}$ between 0.08 and 0.12 ). This may be explained as follows. The $c_{B}^{2}$ for these 7 cases varies from 3.2 to 32.7 ; therefore, both the $F \& B$ and the momentiteration methods do not accurately approximate the variance of the sojourn time. Note that for the cases where $\hat{\sigma}_{L}^{2}$ almost coincides with $\sigma_{L}^{2}, \hat{\rho}_{L}$ also almost coincides with $\rho_{L}$. From these results, we conclude that the performance of the suggested model is moderate. Hence, we recommend to concentrate on the first two moments of the lead time and use the splitting rule of Eq. (8). 
TABLE 1. The (Simulated) Mean, Variance, and Correlation of the Lead Time Observed at a Single Stockpoint, Using the F\&B and de Kok Techniques

\begin{tabular}{lcccccccc}
\hline Policy & Possibility & \multicolumn{1}{c}{$l$} & $\mu_{L}$ & $\hat{\mu}_{L}$ & $\sigma_{L}^{2}$ & $\hat{\sigma}_{\bar{L}}^{2}$ & $\rho_{L}$ & $\hat{\rho}_{L}$ \\
\hline F\&B & 1 & 1.744 & 2.000 & 2.000 & 0.250 & 0.192 & 0.50 & 0.38 \\
F\&B & 2 & 1.315 & 2.000 & 2.002 & 0.250 & 0.208 & 0.50 & 0.42 \\
de Kok & 1 & 1.764 & 2.000 & 1.996 & 0.250 & 0.243 & 0.50 & 0.47 \\
de Kok & 2 & 1.215 & 2.000 & 2.004 & 0.250 & 0.260 & 0.50 & 0.52 \\
F\&B & 1 & 1.863 & 2.000 & 2.002 & 0.250 & 0.195 & 0.65 & 0.53 \\
F\&B & 2 & 1.060 & 2.000 & 2.010 & 0.250 & 0.224 & 0.65 & 0.62 \\
de Kok & 1 & 1.863 & 2.000 & 1.997 & 0.250 & 0.218 & 0.65 & 0.58 \\
de Kok & 2 & 0.967 & 2.000 & 2.018 & 0.250 & 0.297 & 0.65 & 0.70 \\
F\&B & 1 & 1.742 & 2.000 & 2.000 & 0.500 & 0.402 & 0.70 & 0.60 \\
F\&B & 2 & 1.039 & 2.000 & 2.005 & 0.500 & 0.428 & 0.70 & 0.66 \\
de Kok & 1 & 1.742 & 2.000 & 1.995 & 0.500 & 0.439 & 0.70 & 0.63 \\
de Kok & 2 & 0.945 & 2.000 & 2.010 & 0.500 & 0.543 & 0.70 & 0.72 \\
F\&B & 1 & 1.853 & 2.000 & 1.999 & 0.500 & 0.422 & 0.80 & 0.73 \\
F\&B & 2 & 0.841 & 2.000 & 2.017 & 0.500 & 0.468 & 0.80 & 0.78 \\
de Kok & 1 & 1.853 & 2.000 & 1.993 & 0.500 & 0.368 & 0.80 & 0.71 \\
de Kok & 2 & 0.675 & 2.000 & 2.032 & 0.500 & 0.662 & 0.80 & 0.86 \\
F\&B & 1 & 1.673 & 2.000 & 2.000 & 1.000 & 0.825 & 0.80 & 0.73 \\
F\&B & 2 & 0.790 & 2.000 & 2.010 & 1.000 & 0.902 & 0.80 & 0.79 \\
de Kok & 1 & 1.673 & 2.000 & 1.992 & 1.000 & 0.780 & 0.80 & 0.72 \\
de Kok & 2 & 0.669 & 2.000 & 2.021 & 1.000 & 1.157 & 0.80 & 0.83 \\
\hline & & & & & & & & \\
\hline
\end{tabular}

\subsection{Two-Stage Serial System}

Consider the two-serial system of Figure 5. Suppose the splitting rule of Eq. (8) is used in both stockpoints. Stockpoint 1 inspects the inventory at the start of every period (i.e., $R:=1$ ), and upon arrival of such order stockpoint 2 inspects its inventory position and places an order. For stockpoint 1 we have $\mu_{L} \in\{0.5,0.75,1,1.5,2,3\}$ and $c_{L} \in\{0.25,0.5,0.75\}$. For stockpoint 2 we have $\mu_{L}=1$ and $\sigma_{L}^{2}=0.50$. We only varied the lead time parameters of stockpoint 1 , because these influence the behavior of the departure process of stockpoint 1 and, hence, the arrival process of stockpoint 2 . To examine the impact of neglecting the dependency of arrivals at stockpoint 2, we simulated $\hat{\mu}_{L}$ and $\hat{\sigma}_{L}^{2}$ of stockpoint 2 for the aforementioned 18 instances. This was done for the three approximations derived in Buzacott and Shanthikumar [3] (see Table 2). For every instance, we simulated 400,000 periods. Approximations 1 and 2 result in lead times for which both the mean and the variance are too low, whereas approximation 3 generally results in lead times for which both the mean and the variance are too high. It turns out that approximation 1 is always worse than approximation 2. Furthermore, the deviations of the mean lead time of approximations 2 and 3 are almost equal; however, approximation 3 results in a much better 
TABLE 2. The (Simulated) Mean and Variance of the Lead Time Observed at Stockpoint $2\left(\mu_{L}=1\right.$ and $\left.\sigma_{L}^{2}=0.5\right)$, for the Three Approximations of Buzacott and Shanthikumar [4]

\begin{tabular}{|c|c|c|c|c|c|c|c|c|}
\hline & & & \multicolumn{6}{|c|}{ Stockpoint 2} \\
\hline \multicolumn{3}{|c|}{ Stockpoint 1} & \multicolumn{2}{|c|}{ Approximation 1} & \multicolumn{2}{|c|}{ Approximation 2} & \multicolumn{2}{|c|}{ Approximation 3} \\
\hline$\mu_{L}$ & $c_{L}$ & $\rho$ & $\hat{\mu}_{L}$ & $\hat{\sigma}_{\bar{L}}^{2}$ & $\hat{\mu}_{L}$ & $\hat{\sigma}_{L}^{2}$ & $\hat{\mu}_{L}$ & $\hat{\sigma}_{L}^{2}$ \\
\hline \multirow[t]{3}{*}{0.50} & 0.25 & 0.125 & 0.998 & 0.494 & 0.998 & 0.494 & 1.004 & 0.501 \\
\hline & 0.50 & 0.245 & 0.989 & 0.474 & 0.991 & 0.476 & 1.009 & 0.500 \\
\hline & 0.75 & 0.349 & 0.981 & 0.457 & 0.987 & 0.464 & 1.016 & 0.503 \\
\hline \multirow[t]{3}{*}{0.75} & 0.25 & 0.187 & 0.994 & 0.483 & 0.994 & 0.485 & 1.006 & 0.501 \\
\hline & 0.50 & 0.349 & 0.981 & 0.457 & 0.987 & 0.464 & 1.016 & 0.502 \\
\hline & 0.75 & 0.467 & 0.973 & 0.443 & 0.981 & 0.453 & 1.019 & 0.502 \\
\hline \multirow[t]{3}{*}{1.00} & 0.25 & 0.245 & 0.989 & 0.475 & 0.991 & 0.478 & 1.009 & 0.502 \\
\hline & 0.50 & 0.432 & 0.975 & 0.448 & 0.982 & 0.456 & 1.019 & 0.504 \\
\hline & 0.75 & 0.552 & 0.972 & 0.444 & 0.979 & 0.453 & 1.020 & 0.506 \\
\hline \multirow[t]{3}{*}{1.50} & 0.25 & 0.349 & 0.982 & 0.459 & 0.986 & 0.465 & 1.015 & 0.503 \\
\hline & 0.50 & 0.552 & 0.972 & 0.444 & 0.979 & 0.453 & 1.020 & 0.506 \\
\hline & 0.75 & 0.662 & 0.969 & 0.442 & 0.975 & 0.449 & 1.020 & 0.506 \\
\hline \multirow[t]{3}{*}{2.00} & 0.25 & 0.432 & 0.975 & 0.447 & 0.982 & 0.456 & 1.018 & 0.504 \\
\hline & 0.50 & 0.632 & 0.970 & 0.445 & 0.977 & 0.449 & 1.020 & 0.508 \\
\hline & 0.75 & 0.730 & 0.968 & 0.444 & 0.973 & 0.449 & 1.018 & 0.509 \\
\hline \multirow[t]{3}{*}{3.00} & 0.25 & 0.552 & 0.971 & 0.443 & 0.977 & 0.452 & 1.022 & 0.508 \\
\hline & 0.50 & 0.730 & 0.968 & 0.441 & 0.974 & 0.451 & 1.020 & 0.512 \\
\hline & 0.75 & 0.807 & 0.972 & 0.447 & 0.974 & 0.452 & 1.010 & 0.497 \\
\hline \multirow[t]{3}{*}{6.00} & 0.25 & 0.730 & 0.966 & 0.438 & 0.973 & 0.448 & 1.018 & 0.508 \\
\hline & 0.50 & 0.850 & 0.974 & 0.452 & 0.977 & 0.461 & 1.005 & 0.497 \\
\hline & 0.75 & 0.897 & 0.979 & 0.463 & 0.983 & 0.469 & 1.003 & 0.504 \\
\hline
\end{tabular}

performance of the lead time variance. Hence, from this experiment we suggest using approximation 3 . As can be seen from Table 2, the performance of the approximations depends on the utilization, $\rho$ say, of the queue of stockpoint 1 . When $\rho$ is small, customers hardly interact with each other (because almost every customer arrives at an empty queue of stockpoint 1). When $\rho$ is large, almost every customer sees a nonempty queue. Hence, most of the times the interdeparture time of two successive customers equals a service time. Because the service times are independent, the interarrival times at the queue of stockpoint 2 are also independent. Table 2 shows that the performance of the approximations is the worst when $\rho$ is around 0.8 . However, for small $\rho$ and high $\rho$, the approximations yield very satisfactory results.

Finally, we notice that it takes quite some time before the simulator "stabilizes." This issue was already discussed in Vinson [19]. Take for example the instance $\left(\mu_{L}=3\right.$ and $\left.c_{L}=0.75\right)$. Because the parameters of the queuing system of stockpoint 
TABle 3. $\hat{\sigma}_{L}^{2}$ for Several Cycle Times

\begin{tabular}{cc}
\hline Cycle Time & $\hat{\sigma}_{L}^{2}$ \\
\hline 50,000 & 5.655 \\
100,000 & 5.421 \\
200,000 & 5.339 \\
300,000 & 5.273 \\
400,000 & 5.077 \\
\hline
\end{tabular}

1 can be determined by the exact expression of Eq. (9), we know that $\hat{\sigma}_{L}^{2}$ obtained by simulation has to converge to $\sigma_{L}^{2}=5.0625$. In Table 3 , this variance is depicted for several different sizes of the simulation run. That it converges very slowly is due to the high correlation between successive lead times. When this correlation is not so large, the convergence is much quicker.

\section{CONCLUSIONS}

In this paper, we develop a simple model for a lead time process in which orders do not cross. We show that the combination of a single-server queue and a deterministic pipeline yields a versatile and simple lead time process. To model the lead time process for a single location inventory system, we analyzed two methods such that (a) its first two moments match the moments observed in practice and (b) besides its first two moments the first-order correlation also matches. For the first method, exact expressions are derived (Eqs. (8) and (9) for the D/M/1 queue and Eq. (12) for the GI/M/1 queue). For the second method, we developed an algorithm that determines approximate values for the parameters of the queueing model (using Eqs. $(5)-(7),(10)$, and (11)). It turns out that not every coefficient of correlation can be attained. An interesting question, of course, is whether in practice there are lead time processes that do attain such coefficients of correlation. Because in our opinion the performance of the second method is moderate, we recommend using the first method. Furthermore, the results are extended to arbitrary large divergent multi-echelon systems. Now the performance of the first method depends on the impact of neglecting the dependency between arrivals (if there is any). Numerical results are quite satisfying (see Table 2).

\section{References}

1. Adan, I., van Eenige, M., \& Resing, J. (1995). Fitting discrete distributions on the first two moments. Probability in the Engineering and Informational Sciences 9: 623-632.

2. Anupindi, R., Morton, T.E., \& Pentico, D. (1996). The nonstationary stochastic lead-time inventory problem: Near-myopic bounds, heuristics, and testing. Management Science 42: 124-129.

3. Bhat, V.N. (1993). Approximation for the variance of the waiting time in a GI/G/I queue. Microelectronics and Reliability 33: 1997-2002.

4. Buzacott, J.A. \& Shanthikumar, J.G. (1993). Stochastic models of manufacruring systemis. Englewood Cliffs, NJ: Prentice-Hall. 
5. Ehrhardt, R. (1984). $(s, S)$ policies for a dynamic inventory model with stochastic lead time. Operations Research 32: 121-132.

6. Fredericks, A.A. (1982). A class of approximations for the waiting time distribution in a GI/G/1 queueing system. Bell System Technical Journal 61: 295-325.

7. Friedman, M.F. (1984). On a stochastic extension of the EOQ formula. European Journal of Operational Research 17: 125-127.

8. Gross, D. \& Soriano, A. (1969). The effect of reducing leadtime on inventory levels-simulation analysis. Management Science 16: B61-B76.

9. Hadley, G. \& Whitin, T.M. (1963). Analysis of inventory systems. Englewood Cliffs, NJ: PrenticeHall.

10. Heijden, M.C. van der, Diks, E.B., \& de Kok, A.G. (1996). Stochastic lead times in multi-echelon divergent systems. Memorandum COSOR gb-36, Eindhoven University of Technology, Eindhoven, The Netherlands.

11. Heuts, R. \& de Klein, J. (1995). An $(s, q)$ inventory model with stochastic and interrelated lead times. Naval Research Logistics 42: 839-859.

12. Kaplan, R.S. (1970). A dynamic inventory model with stochastic lead times. Management Science 16: 491-507.

13. Kok, A.G. de (1989). A moment-iteration method for approximating the waiting-time characteristics of the GI/G/l queue. Probability in the Engineering and Informational Sciences 3: 273-287.

14. Krämer, W. \& Langenbach-Belz, M. (1978). Approximate formulae for the delay in the queueing system GI/G/1. Proceedings of the 8th International Teletraffic Congress, Melbourne, 235-1/8.

15. Nahmias, S. (1979). Simple approximations for a variety of dynamic leadtime lost-sales inventory models. Operations Research 27: 904-924.

16. Sphicas, G.P. (1982). On the solution of an inventory model with variable lead times. Operations Research 30: 404-410.

17. Sphicas, G.P. \& Nasri, F. (1984). An inventory model with finite-range stochastic lead times. Naval Research Logistics Quarterly 31: 609-616.

18. Tijms, H.C. (1986). Stochastic modelling and analysis: A computational approach. Chichester: John Wiley \& Sons.

19. Vinson, C.E. (1972). The cost of ignoring lead time unreliability in inventory theory. Decision Sciences 3: 87-105.

20. Whitt, W. (1982). Refining diffusion approximations for queues. Operations Research Letters 1: 165-169.

21. Yano, C.A. (1987). Stochastic leadtimes in two-level distribution-type networks. Naval Research Logistics 34: 831-843.

22. Zipkin, P. (1986). Stochastic leadtimes in continuous-time inventory models. Naval Research Logistics Quarterly 33: 763-774. 


\section{APPENDIX}

Nested Bisection Algorithm to Determine $\mu_{B}$ and $\sigma_{B}^{2}$ Such That the Sojourn Time Has

Mean $\mu_{S}$ and Variance $\sigma_{S}^{2}$

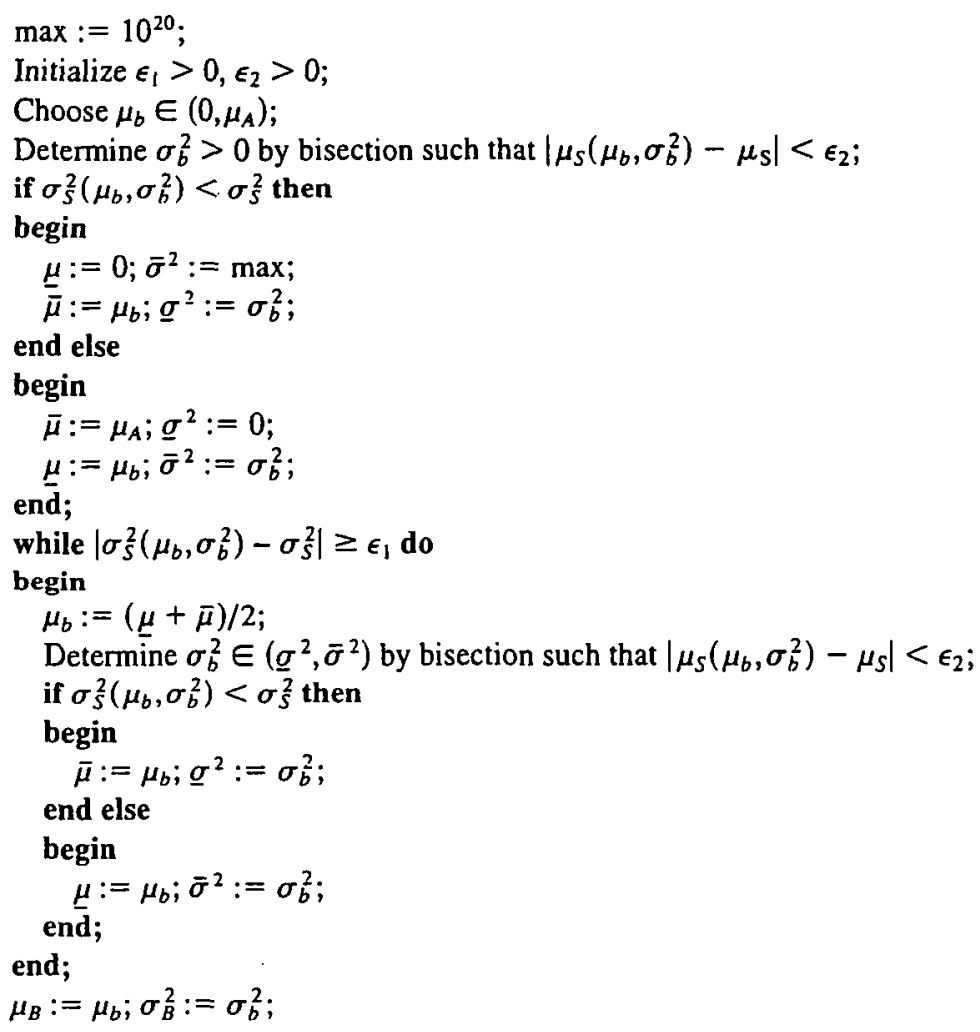

